\title{
Comparison of Methods for the Simplification of Mesh Models using Quality Indices and an Observer Study
}

\author{
Samuel Silva, ${ }^{a}$ Joaquim Madeira, ${ }^{a, b}$ Carlos Ferreira ${ }^{c, d}$ and Beatriz Sousa Santos ${ }^{a, b}$ \\ ${ }^{a}$ Instituto de Engenharia Electrónica e Telemática de Aveiro, Universidade de Aveiro, Portugal \\ ${ }^{b}$ Dep. Electrónica, Telecomunicações e Informática, Universidade de Aveiro, Portugal \\ ${ }^{c}$ Dep. Economia, Gestão e Engenharia Industrial, Universidade de Aveiro, Portugal \\ ${ }^{d}$ Centro de Investigação Operacional, Universidade de Lisboa, Portugal
}

\begin{abstract}
The complexity of a polygonal mesh model is usually reduced by applying a simplification method, resulting in a similar mesh having less vertices and faces. Although several such methods have been developed, only a few observer studies are reported comparing them regarding the perceived quality of the obtained simplified meshes, and it is not yet clear how the choice of a given method, and the level of simplification achieved, influence the quality of the resulting model, as perceived by the final users. Mesh quality indices are the obvious less costly alternative to user studies, but it is also not clear how they relate to perceived quality, and which indices best describe the users behavior.

Following on earlier work carried out by the authors, but only for mesh models of the lungs, a comparison among the results of three simplification methods was performed through (1) quality indices and (2) a controlled experiment involving 65 observers, for a set of five reference mesh models of different kinds. These were simplified using two methods provided by the OpenMesh library - one using error quadrics, the other additionally using a normal flipping criterion - and also by the widely used QSlim method, for two simplification levels: 50\% and $20 \%$ of the original number of faces. The main goal was to ascertain whether the findings previously obtained for lung models, through quality indices and a study with 32 observers, could be generalized to other types of models and confirmed for a larger number of observers. Data obtained using the quality indices and the results of the controlled experiment were compared and do confirm that some quality indices (e.g., geometric distance and normal deviation, as well as a new proposed weighted index) can be used, in specific circumstances, as reasonable estimators of the user perceived quality of mesh models.
\end{abstract}

Keywords: Meshes, perceived quality, evaluation, user study, mesh simplification.

\section{INTRODUCTION}

Polygonal meshes are widely used to represent different kinds of models in many application domains (e.g., medical visualization, CAD/CAM, physically-based modeling and computer games). Whenever such models are defined by a relatively large number of faces, and due to insufficient memory or low processing power, the required rendering time might not be adequate to allow interactive visualization, a mostly desirable feature in, for instance, decision scenarios, where the choice of adequate views is important. In this case, a possible solution consists in showing a simplified version while the user interactively chooses the viewpoint and, then, a fully detailed version of the model to support its analysis. To tackle this problem, simplification methods can be used to generate a similar and more manageable, less complex mesh model having a smaller number of vertices and faces.

While several such simplification methods have been developed and are reported in the literature (e.g., see the survey by Luebke ${ }^{1}$ ), only a few evaluation studies are reported comparing them regarding the perceived quality of the obtained simplified meshes. ${ }^{2}$ Moreover, it is not yet clear how the choice of a given method, and the level of simplification achieved, influence the quality of the resulting model, as perceived by the final users. Clearly, this should be taken into account when choosing a particular simplification method for a given application domain.

Send correspondence to Samuel Silva (sss@ieeta.pt). 

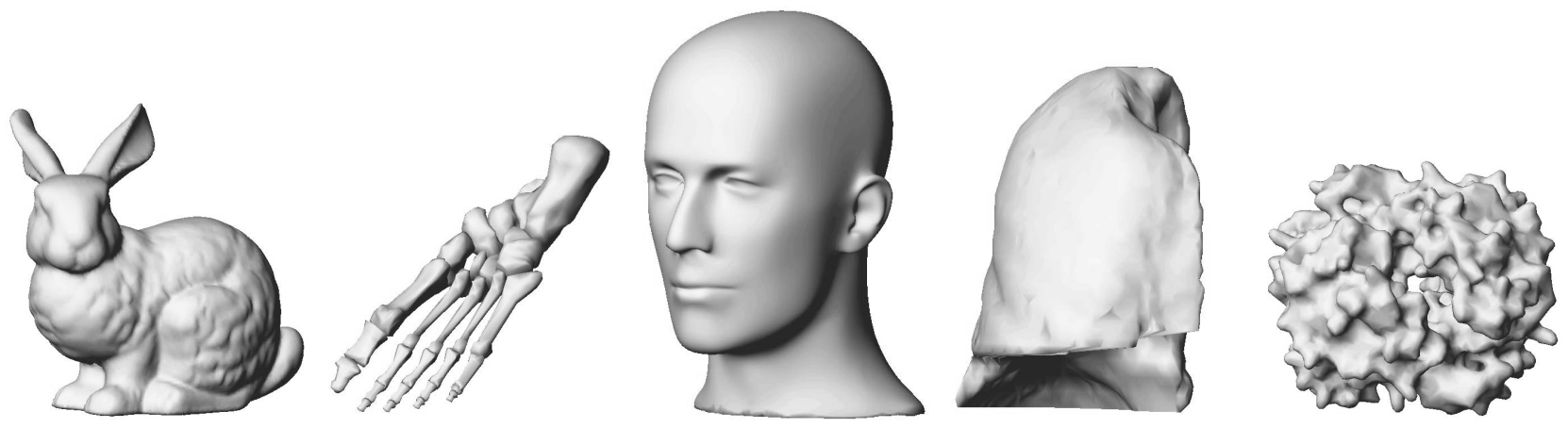

Figure 1. Original models: Bunny, Foot, Head, Lung and Strange (from left to right).

Applying any simplification method to a polygonal mesh model entails the existence of differences (i.e., errors or deviations) between the original and the resulting simplified mesh. There are tools ${ }^{3-5}$ which allow computing some quality indices (e.g., geometric distance and minimum angle), which have been used to perform quality evaluation after mesh processing operations. Although such data allows quantifying the difference between meshes representing the same model, it is not yet clear which indices best describe, and to what degree, the user perceived quality of simplified meshes.

Thus, to assess the relative performance of mesh simplification methods, user perceived mesh quality still has to be evaluated through observer studies. Even though they provide a ground truth, such user studies do require a large amount of resources (e.g., number of participants and time spent). Appropriate mesh quality indices are the obvious, less costly alternative to observer studies, but, as stated, it is not clear how they relate to user perceived quality. This can only be achieved by understanding how mesh quality indices, computed for a generic set of test models, relate with the results of an observer study carried out, for the same set of models, in order to evaluate user perceived mesh quality.

Following on earlier work carried out by the authors, at the time only for mesh models of the lungs, ${ }^{6}$ a comparison among the results of three simplification methods was performed through (1) quality indices and (2) a controlled experiment involving 65 observers, for a set of five reference mesh models of different kinds (see Fig. 1). These were simplified using two methods provided by the OpenMesh library ${ }^{7}$ — one using error quadrics, the other additionally using a normal flipping criterion - and also by the widely used $Q$ Slim method, ${ }^{8}$ for two simplification levels: $50 \%$ and $20 \%$ of the original number of faces (one of the test model sets is shown in Fig. 2). A separate data analysis of the computed quality indices and the results of the observer study was performed first; afterwards, the global results were compared to try to understand how quality indices relate to user perceived mesh quality. The main goal was to ascertain whether the findings that had been obtained for lung models, through quality indices and a study with 32 observers, could be generalized to other types of models and confirmed for a larger number of observers, thus becoming useful guidelines for practitioners. We also intended to evaluate a weighted quality index based upon those findings.

In the following section, the main findings obtained earlier for lung models are mentioned, in order to allow an easy comparison with the results presented here for generic models. After presenting the set of test models used throughout, the evaluation data obtained using different mesh quality indices is analyzed in detail, in the next section. The observer study and its main results are presented afterwards. Finally, a comparative analysis of the global results is done and some conclusions presented.

\section{PREVIOUS WORK}

The need for observer studies to evaluate the outcome of new techniques has again been recently emphasized for the Visualization area. ${ }^{9}$ Resulting from the knowledge and insight gained from adequate evaluation studies, it should be possible to establish guidelines that can help Visualization and Geometric Modeling practitioners choose which mesh simplification methods to use in different circumstances. In addition, it is also desirable to 

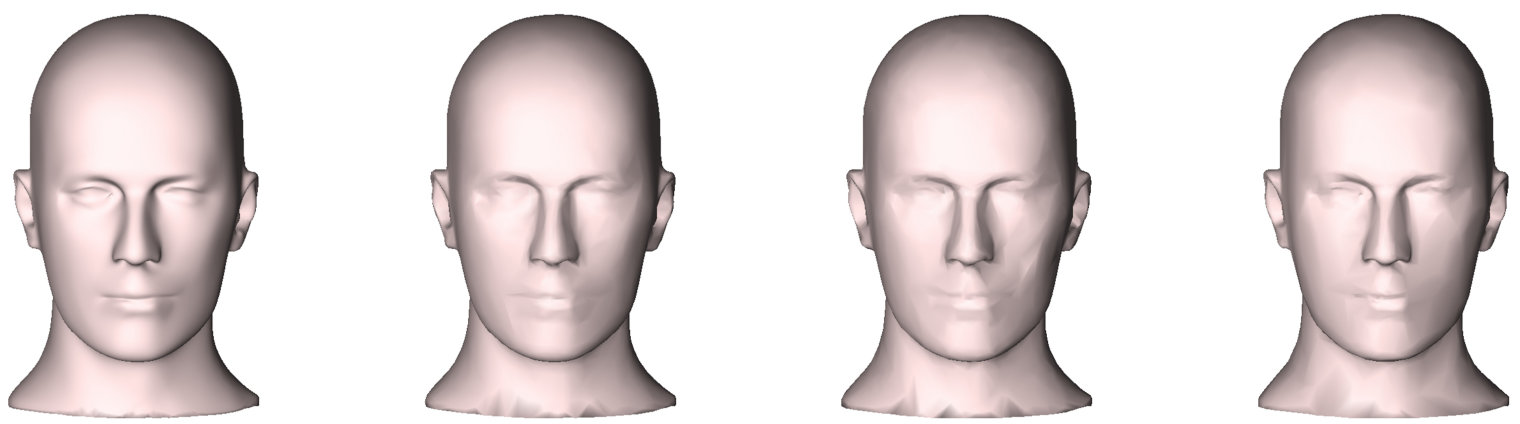

Figure 2. Sample test model set: original Head model and three severely simplified versions using QSlim and the two methods provided by the OpenMesh library (from left to right).

identify which quality indices are the most appropriate (in the sense that they estimate the users behavior) for evaluating the results of the simplification operations.

Aiming at establishing guidelines for the proper choice of mesh simplification methods, the authors have been evaluating the quality of simplified mesh models both using observer studies and computing quality indices. Our first objective was to try to find out which methods entail better user perceived quality for the same simplification level. Since such observer studies are costly, we have also been studying quality indices which might provide an alternative measure of user perceived mesh quality, and be reasonable estimators of the users preferences.

The whole research work carried out is based on three simplification methods deemed representative: the widely used QSlim $^{8}$ and the other two provided by the relatively recent OpenMesh ${ }^{7}$ library — one using error quadrics, the other additionally using a normal flipping criterion. Two simplification levels were set: moderate simplification to $50 \%$ and severe simplification to $20 \%$ of the number of original model faces. In a first stage, we dealt only with mesh models of the lungs; currently, we are using generic mesh models of different kinds, and investigating whether the particular earlier findings are confirmed for such models.

As thoroughly described by Silva et al., ${ }^{10}$ an experimental framework, supporting software, and a data analysis methodology were developed which allowed conducting a users study with 32 observers, who evaluated, for the three methods and simplification levels chosen and by performing preference and rating tasks, 24 simplified lung models according to their perceived quality. A within subjects experimental design was used and all participants could interact (e.g., zoom, rotate) with each 3D model at all experimental conditions, although in different order.

The analysis of the collected data suggested that the simplification level does influence the observers' decision times, as expected from the onset of the experiment: they seem to make faster decisions for the severely simplified models (20\%). More important, observers seem also to be responsive to the simplification method used, although they react differently according to the simplification level: for a moderate simplification (50\%), the models generated by the OpenMesh simplification method, with the additional normal flipping criterion, obtained the best results for the preference task; for a severe simplification, it is the QSlim method that generates most of the models classified in the first place, this time for both tasks.

In Santos et al. ${ }^{6}$ we used several quality indices to analyze the differences between the original and the simplified versions of all the lung models used in the previous study: for each one of the 24 simplified models, we computed six quality indices regarding the corresponding original. Namely, we determined the maximum, mean and variance values of the geometric distance (the distance between corresponding points on both surfaces), as well as of the normal deviation (the difference between corresponding normal vectors).

A preliminary analysis of the data showed that, for the three geometric distance quality indices, QSlim is the best classified method for both simplification levels. However, for the three normal deviation indices, the best classified method is the one provided by OpenMesh, with the additional normal flipping criterion. Further analysis regarding the mean geometric distance values showed that, for both simplification levels, QSlim is the best method, followed by the OpenMesh simplification method using error quadrics, and by the OpenMesh 

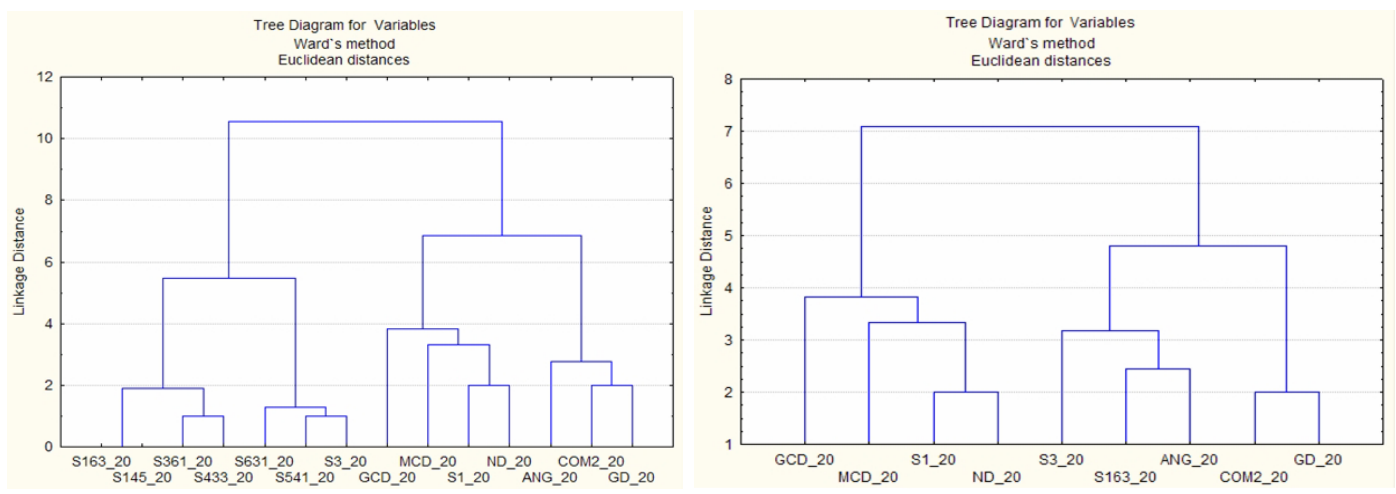

Figure 3. Left, dendogram showing associations between all computed quality indices for severe simplifications; right, dendogram showing associations after discarding some redundant quality indices.

method using the additional normal flipping criterion. For the mean normal deviation no clear association could be established for the severe (20\%) simplification level, while for moderately simplified models the OpenMesh simplification method, with the normal flipping criterion, was the best classified, followed by QSlim, and by the OpenMesh method using error quadrics.

Additional comparison between those results and the ones obtained for the previous observer study showed that, at least in those specific circumstances, the mean geometric distance between the original and the simplified mesh could be a good estimator of the observers' preferences for severely (20\%) simplified models, but not for moderate simplifications. Conversely, mean normal deviation might be a good estimator of the observers' preferences for moderately (50\%) simplified models.

The findings briefly presented above suggest that users might be more sensitive to the geometric distance, between a simplified model and its original, in the presence of less information, and more sensitive to the normal deviation in the presence of more information (i.e., for moderately simplified models). Nevertheless, this result has to be confirmed by an observer study involving more observers, as well as generalized to other kinds of models. Other quality indices should also be considered. Such are the objectives of the work reported in the remaining of this paper.

\section{QUALITY INDICES}

Quality indices have been used in many situations to assess the resulting quality of several mesh processing methods. Some tools like Metro, ${ }^{3}$ MeshDev $^{4}$ and more recently PolyMeCo, ${ }^{5}$ the mesh analysis and comparison tool being developed at the University of Aveiro, allow the computation of various quality indices for polygonal mesh models.

In what follows the quality assessment of several simplified mesh models is described. First, a short presentation of the evaluated model set is provided. Then, the quality indices, which were computed using PolyMeCo, are briefly described. Finally, the analysis of the mean values obtained for each quality index is presented.

\subsection{Simplified Mesh Models}

In order to be able to compare the outcomes of the observer study, and the analysis using quality indices, with the findings previously obtained for mesh models of the lungs, test models were generated using the same methods (from now on denoted simply by $Q S, O M$ and $O M N F$ ) and simplification levels (50\% and 20\%) as before.

Model sets were built from the set of five reference models (see Fig. 1): for each model and each simplification level three simplified models were created using the three simplification methods. This resulted in a total of 10 test sets, each composed by an original and three simplified models (see Fig. 2, for an example of such a set). Note that the five original, reference models chosen are different from each other and have different numbers of vertices and faces. 

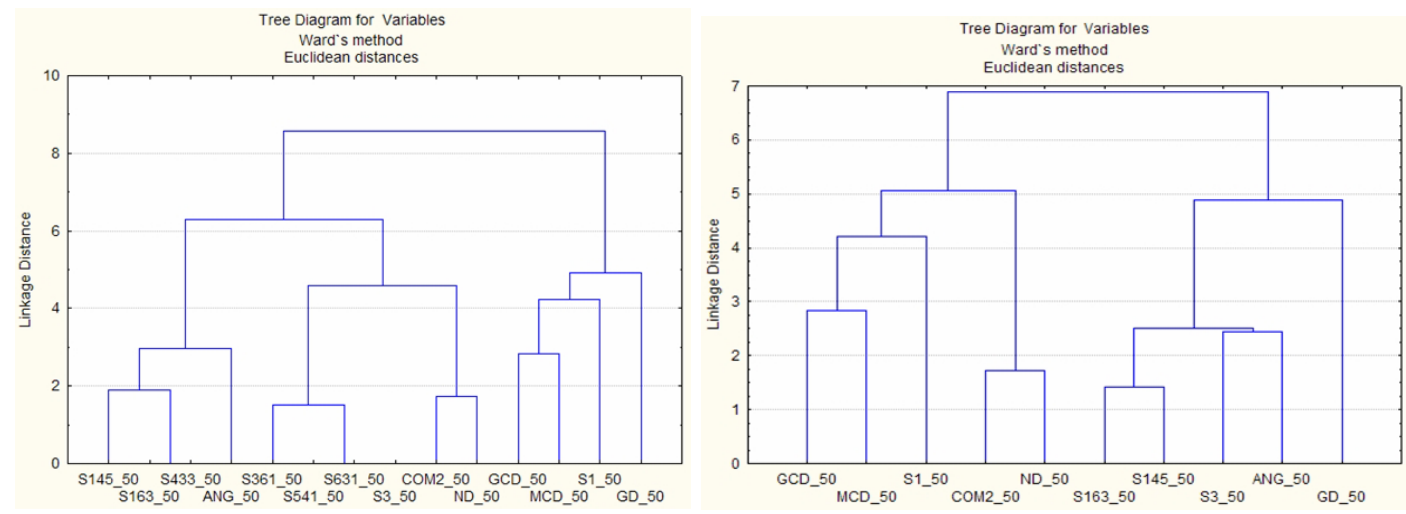

Figure 4. Left, dendogram showing associations between all computed quality indices for moderate simplifications; right, dendogram showing associations after discarding some redundant quality indices.

\subsection{Quality Indices}

Several quality indices provided by PolyMeCo ${ }^{5}$ were computed for each one of the 30 simplified models:

- Geometric Distance (GD) - This quality index computes the distance between corresponding points of two model surfaces; ${ }^{4}$

- Normal Deviation (ND) - This quality index measures the deviation between surface normals of two models. This can be an important issue, since surface normals are directly related with the rendering process: a strong deviation may result in visual artifacts; ${ }^{4}$

- Composed Deviation (COM2) - This quality index is being proposed based upon our previous results which suggest that the Geometric Distance is a good estimator of user perceived quality for severe simplifications, while Normal Deviation is a good estimator for moderate simplifications. Thus, these two quality indices are weighted according to the simplification level of the mesh;

- Smoothness - To measure smoothness some alternatives were considered. S3 is the usual smoothness measure which computes the distance of each vertex to the centroid of its one ring. S1 computes smoothness using, not the norm of the vertex-centroid distance, but the difference vector, which implies that, when computing the mean value, symmetric differences will cancel each other. This tries to explore the fact that, if a model has a less smooth surface, it can still be deemed acceptable if it maintains some symmetry. Finally, the $\mathbf{S} i j k$ indices compute smoothness using vertices from the first, second and third neighborhoods of a vertex, assigning them weights of $0.1 \times i, 0.1 \times j$ and $0.1 \times k$, respectively;

- Mean and Gaussian Deviations (MCD, GCD) - These indices express the differences in Mean and Gaussian curvature between two models; ${ }^{11}$

- Angle Analysis (ANG) - This index computes the smallest angle for each triangle which composes the mesh. A minimum angle close to 60 degrees identifies a triangle close to equilateral; a smaller value identifies deformed triangles (e.g., flat or needle triangles). ${ }^{12}$

Note that PolyMeCo was used to compute the values distribution for each simplified model and quality index, but only the mean values (deemed more representative) were considered for the data analysis.

\subsection{Data Analysis}

For every simplified model, the mean value for each one of the above described quality indices was computed. Based upon those mean values, for each simplified model and for the same simplification level, a rank (first, second and third place) was assigned to the three models comprising each test set. 

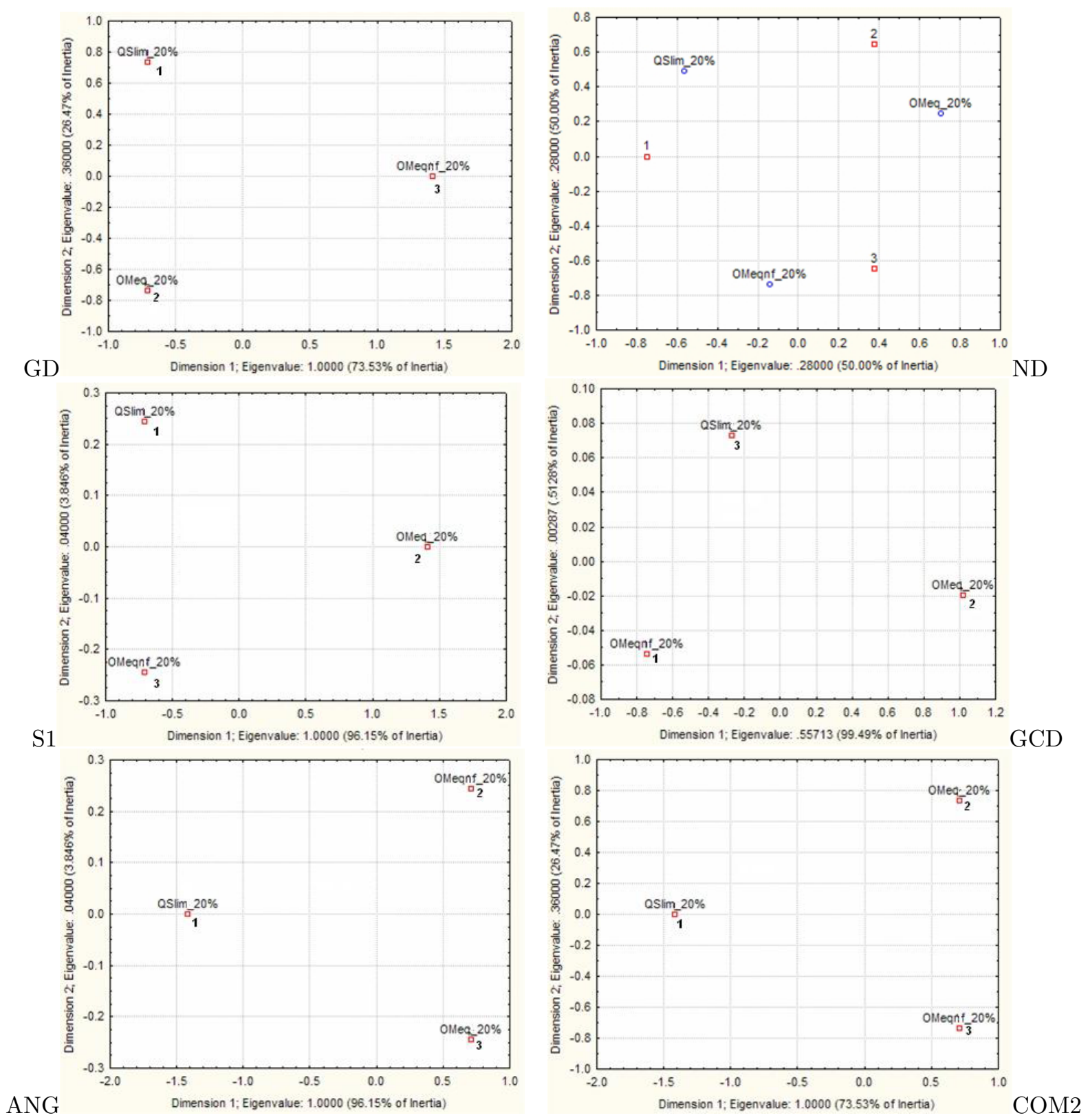

Figure 5. Quality indices: correspondence analysis showing the associations between simplification method and ranks obtained for severe simplification. 

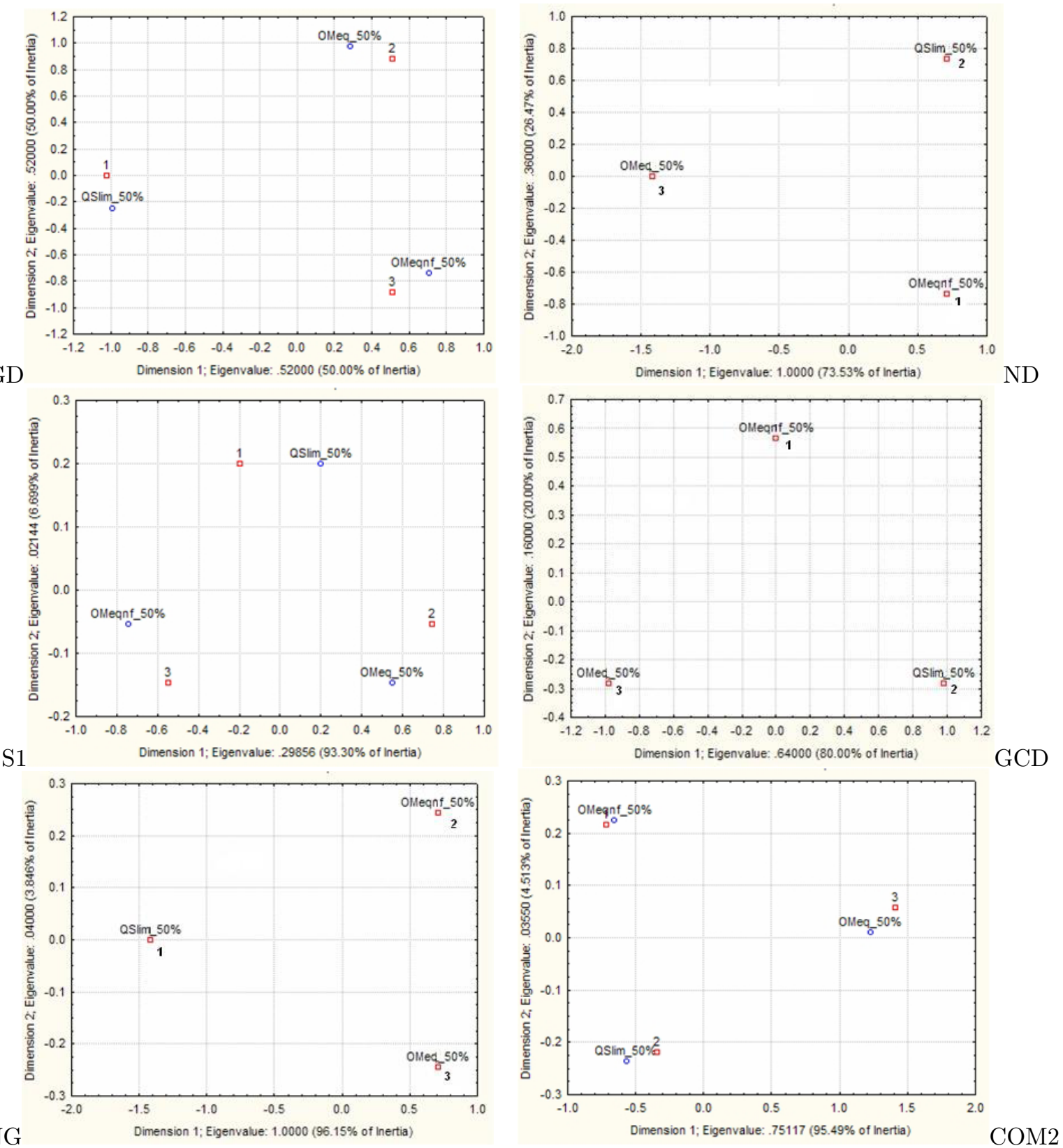

Figure 6. Quality indices: correspondence analysis showing the associations between simplification method and ranks obtained for moderate simplification. 
To study the association between quality indices, and to assert possible redundancies in the results provided by different indices, Cluster Analysis ${ }^{13}$ was used. For severely simplified (20\%) models, and looking at the dendogram on the left of Fig. 3 (using Ward's method as a measure of proximity), it is possible to detect some associations between indices, namely across the Sijk group. A closer look at the data revealed some degree of redundancy. After discarding the indices deemed redundant, a new dendogram was computed (Fig. 3 on the right), which is now easier to read. Notice that, as it was expected, the COM2 index appears associated with the GD for the severe simplifications. Notice also that S1 and S3 appear clearly separated in different branches.

For the moderate simplification a similar analysis was performed. To detect possible redundancies, Cluster Analysis was used and a dendogram was drawn for all indices (Fig. 4, on the left). Once again some redundancy was detected and after analyzing the obtained data, several indices were discarded and a new dendogram was drawn (Fig. 4, on the right). Notice that, as expected, the COM2 index now appears associated with the ND.

After this preliminary analysis, contingency tables were built for the results obtained with each quality index and for each simplification level. The results were then visualized using Correspondence Analysis. ${ }^{13}$ Some of the resulting factorial planes are presented in Fig. 5, for the severely (20\%) simplified models, and in Fig 6, for moderately $(50 \%)$ simplified models.

Regarding the global performance of the quality indices, different behaviors can be identified from Figs. 5 and 6. Namely, for the GD index and for both simplification levels, the associations between method and rank are clearly presented: $Q S$ is associated with the first place, followed by $O M$ in second, and $O M N F$ in the third place, which indicates that $Q S$ is the simplification method producing, in general, simplified models with lower geometric distance to the original reference models, followed by $O M$ and $O M N F$. For severe $(20 \%)$ simplifications, ND does not present such a clear association, although that happens for moderate simplifications; a similar situation occurs with S1, but for the the $50 \%$ and $20 \%$ simplification levels, respectively.

Note that, for severe simplifications, for indices such as GCD and ANG there is also a clear method and rank association, but different from the association found for GD and COM2. The latter two quality indices seem to have a similar behavior, and are able to clearly discriminate between the three simplification methods (see Fig. 5); as said before, this association is also clearly shown in the right dendogram of Fig. 3.

A similar analysis can be done for the 50\% simplification level, using the factorial planes depicted in Fig. 6 . It is possible to verify, for example, clear associations between method and rank for the ND and COM2 indices, with $O M N F$ associated with the first place, $Q S$ with second and $O M$ with the third place. For indices such as GD and GCD there are clear but different method and rank associations. Note that the ND and COM2 quality indices seem to have now a similar behavior, and are able to clearly discriminate between the three simplification methods; as said before, this association is also clearly shown in the right dendogram of Fig. 4.

The main conclusions that can already be mentioned are the following: GD is a quality index clearly able to discriminate between the three simplification methods but for severe simplifications only, while ND plays the same role but for moderate simplifications only; this is the behavior that had already been identified for those same quality indices when analyzing lung models; ${ }^{6}$ the composed quality index COM2, which was established based on that previous study, is able to discriminate between the three simplification methods for both simplification levels, as was expected. Note that, since the behavior of the GD and ND quality indices is globally the same for the lung models of the former study, and for the generic models of the current study, it seems that simplification method and level are the determinant factors, not the type of models being simplified.

\section{OBSERVER STUDY}

The observer study was set up and carried out exactly as it had been done for the previous study using lung models (see Silva et al. ${ }^{10}$ for a thorough description of the objectives, context, framework, experimental methodology and data analysis of that former study), which provided a suitable testbed to confirm that the developed experimental design and protocol allowed the evaluation of user perceived model quality, as well as to establish the methodology for the statistical analysis of the collected data.

To allow an easy implementation of the experimental protocol, as well as easy storage and management of the collected data, the same software application that had been developed for the former lung models study was also 

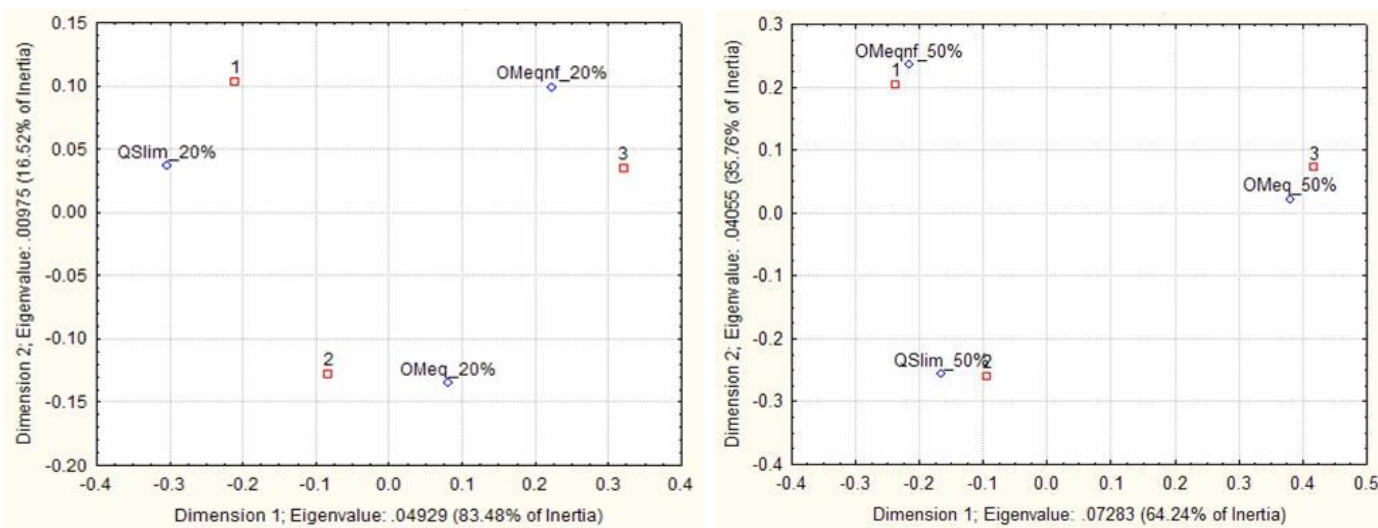

Figure 7. Observer study: correspondence analysis, showing the associations between simplification methods and observer preferences, for severe (left) and moderate (right) simplifications.

used. Note that observers were freely allowed to interact with any model, by changing its position, orientation and scaling factor, and choose the viewpoints they wished to analyze a model from, which is a more realistic setting than the one used by Watson et al. in a similar study. ${ }^{2}$

\subsection{The Experiment}

To assess the hypothesis that distinct mesh simplification methods have different effects on the model quality perceived by human observers, possibly varying with the simplification level and other factors, we asked for the observers' preferences and ratings, which are widely used to obtain relative judgments from observers and seem to be the most adequate indices of fidelity. ${ }^{2}$

A within subjects experimental design was used, i.e., each observer performed under each different condition. Due to the possible influence of learning effects, nervous behavior in the first task or fatigue in the last, all test sets were presented randomly to each observer and, for each observer, models were presented in random order within each set.

For each observer, the experiment was divided into two phases:

In the first phase - preference task - , an observer was sequentially presented with each one of the 10 test sets and asked to assign an ordering (first, second and third place) to the three simplified models, according to their perceived quality regarding the original reference model.

In the second phase - rating task - , an observer was sequentially presented with an original model and one of its simplified versions, taken from one of the test sets, and asked to classify the simplified model using a five level Likert scale ${ }^{14}$ from 1 (very bad) to 5 (very good), once again based on its perceived quality.

Note that the time taken to reach a decision and the number of interactions (performed on each model before deciding) were recorded for each task, since they seemed to be related to the degree of difficulty observers encounter in performing the preference and rating tasks.

Sixty-five engineering students and lecturers, aged between 18 and 55 (the majority, 45 participants, was between 18 and 25 years old), participated in the experiment (57 men and 8 women); 41 participants declared to have experience in viewing/manipulating 3D models. Since the gender, age or experience with 3D object manipulation of an observer might influence the results, this information was used to characterize the profile of each observer.

\subsection{Data Analysis}

The main results obtained from the analysis of the collected data are presented in what follows. For a more detailed analysis, see the paper by Silva et al. ${ }^{15}$ 
An Exploratory Data Analysis ${ }^{16}$ (EDA) provided general information on the structural relations, showing the amplitudes, asymmetries, localizations, outliers, etc. of the collected data. In addition, we used ANOVA to test for the equality of means, Contingency Tables to test the independence between variables, Correspondence Analysis ${ }^{13}$ to reveal possible associations between variables, and Cluster Analysis ${ }^{13}$ to find out similarities among variables. All results were obtained using Statistica. ${ }^{17}$

Concerning the observer preferences, and for severely simplified models (20\%), a tendency was found of the observers to prefer the simplified models using the QSlim method $(Q S)$, then the versions simplified by the OpenMesh method $(O M)$ and, in third place, the versions simplified using the OpenMesh method with the additional normal flipping criterion $(O M N F)$. Contrarily, for moderately simplified models $(50 \%)$, the tendency found was for observers to prefer first the models simplified by the $O M N F$ method, followed by those simplified by QSlim, and the models simplified by the $O M$ method coming in third place.

Contingency tables were used and independency hypothesis were tested to confirm the statistical significance of those two tendencies: the independency between the simplification method and the observer preferences was rejected for both simplification levels, which suggests that observers are indeed responsive to the simplification method used, although they react differently for each simplification level. The results obtained by the contingency tables can be visualized using a Correspondence Analysis: Fig. 7 shows the factorial planes corresponding to the contingency tables for both simplification levels. For the severe simplification level (20\%), we can see that each simplification method is clearly associated to a type of preference: first place for $Q S$, second for $O M$ and third for $O M N F$. A different and even stronger association appears for moderately simplified (50\%) models: the $O M N F$ method is associated with the first place, $Q S$ with the second and $O M$ with the third.

Concerning the observer ratings, a tendency was found of the observers to rate poorly all the severely simplified models (specially those simplified with the $O M N F$ method), while assigning higher ratings to the moderately simplified models (all methods seem equally well rated, perhaps with a slight advantage of the OMNF method). Again, in order to confirm statistical significance, contingency tables were used and independency hypothesis tested, confirming that observers are responsive to both simplification method and level.

The results obtained regarding observer preferences, consistently confirmed applying different statistical methods, suggest that observers are indeed responsive to the simplification method used, although they react differently to the chosen simplification level. Contrarily, results obtained for the rating task seem to imply that observers are more influenced by the simplification level than by the simplification method.

The global findings of this experiment confirm those previously obtained for the observer study using only lung models: for each simplification level, each simplification method obtained similar preferences and ratings. Thus, the different nature of the models used in this later study did not seem to affect the results in a significant way. For a more detailed analysis and comparison of the collected data see the former paper by Silva et al. ${ }^{15}$

\section{COMPARISON OF THE GLOBAL RESULTS}

As the used quality indices provide information about differences between two meshes (a clue towards the quality of the simplified models), we compared the results thus obtained with those provided by the observer study, and tried to identify the quality index which better estimates model quality, as it is perceived by users.

By comparing the factorial plane on the left of Fig. 7, showing the associations between simplification method and observer preferences for the severe simplifications, with the factorial planes of Fig. 5 we find some similarities of association in all presented quality indices except for ANG and GCD. Note that the ND index exhibits a weaker association. On the other hand, by comparing the factorial plane on the right of Fig. 7 with those of Fig. 6 , obtained for moderate simplifications, some similar associations are observed for the ND, GCD and COM2 indices.

These comparison results suggest that, for example, the GD index might be a good estimator of user perceived quality for severe simplifications, but failing for moderate simplifications where the ND or GCD indices seem more appropriate. These results are similar to those obtained in the previous study for lung models. The COM2 index seems to be a good estimator of user perceived quality for both simplification levels ranking all methods the same way users did. It was an expected result since it blends both GD and ND indices, assigning a larger weight to ND when models are moderately simplified and to GD when in the presence of severe simplifications. 

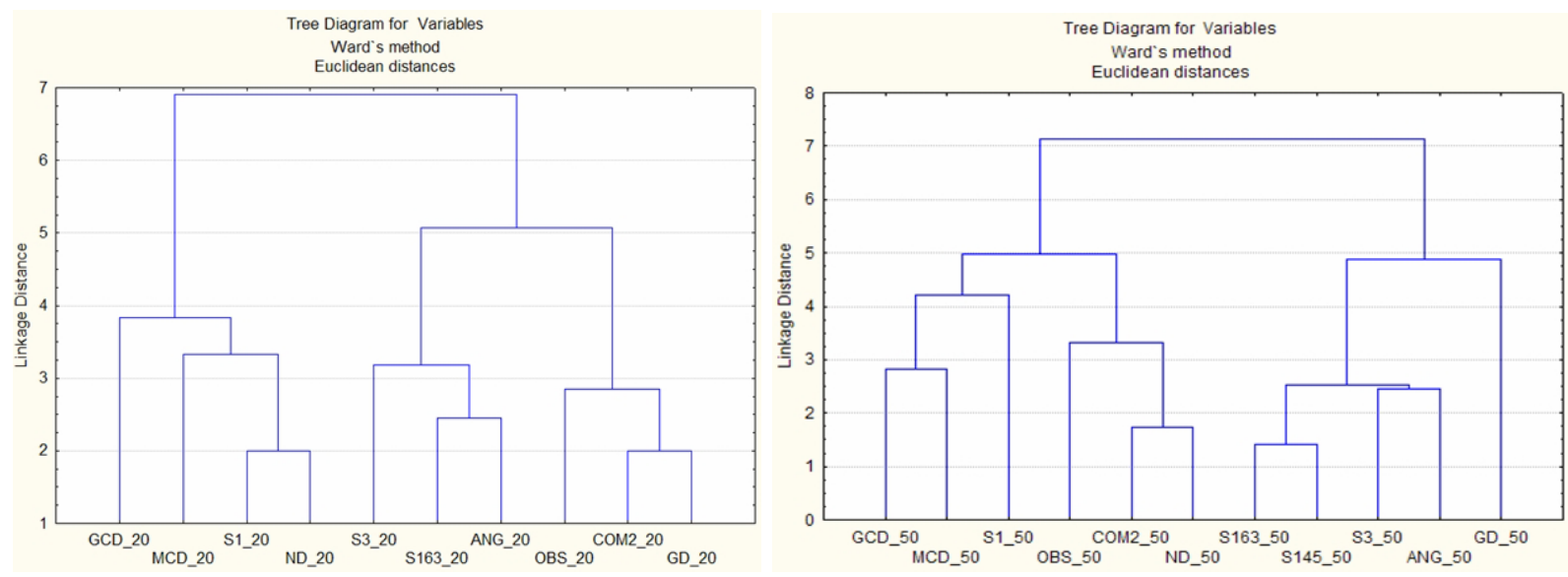

Figure 8. Dendograms (left, severe simplification; right, moderate simplification) showing the association between quality results obtained using the observer study and quality indices.

To confirm these findings Cluster Analysis was once again used. The results obtained in the observer study were treated like a quality index and dendograms were drawn, depicting the associations between all indices. For the severe simplifications (Fig. 8, on the left) the observer (OBS) results appear associated with COM2 and GD; for the moderate simplifications (Fig. 8, on the right) the observer results appear associated with COM2 and ND.

\section{CONCLUSIONS}

We have compared three mesh simplification methods at two simplification levels. This comparison was performed through two different approaches: an observer study and objective quality indices. While the former approach has the advantage of being capable of producing a "golden standard", it is very expensive in terms of several resources. That is why it would be very interesting to identify quality indices capable of reasonably estimating the observers' behavior. Yet, quality indices usually don't correlate well with the results obtained through observer studies.

We selected several quality indices in order to assess if any of them might be such a good estimator of the observers' behavior, at least in some specific circumstances. We confirmed a result previously presented in Santos et al. ${ }^{6}$ suggesting the Geometric Deviation as a good estimator for severe simplifications and the Normal Deviation as a good estimator for moderate simplifications. This fact had already been taken into account when developing the Composed Deviation quality index (COM2), which was the best estimator for both simplification levels.

Note that the smoothness indices, computed using several neighborhoods, did not provide useful results: only S1 seemed to estimate user performance for severe simplifications. Among the curvature deviations, the Gaussian Curvature Deviation seemed a good estimator of user performance for moderate simplifications. Angle Analysis did not provide any significant results.

Clearly these results do require further confirmation. The results provided by the Composed Deviation were obtained for two simplification levels deemed representative, but how will it behave for a wider range of simplification levels? To answer this question we intend to perform further work in order to understand how the observer perceived quality varies across simplification levels and which adjustments are needed in the Composed Deviation index(e.g., the blending factor) to encompass such changes.

\section{ACKNOWLEDGMENTS}

The authors are grateful to all participants in the observer study.

The first author would like to thank research unit 127/94 IEETA of the University of Aveiro for the grant that supports his work. 


\section{REFERENCES}

1. D. Luebke, "A developer's survey of polygonal simplification algorithms," IEEE Computer Graphics and Applications 21(3), pp. 24-35, 2001.

2. B. Watson, A. Friedman, and A. McGaffey, "Measuring and predicting visual fidelity," in Proc. SIGGRAPH 2001, pp. 213-220, 2001.

3. P. Cignoni, C. Rocchini, and R. Scopigno, "Metro: measuring error on simplified surfaces," Computer Graphics Forum 17(2), pp. 167-174, 1998.

4. M. Roy, S. Foufou, and F. Truchetet, "Mesh comparison using attribute deviation metric," International Journal of Image and Graphics 4(1), pp. 1-14, 2004.

5. S. Silva, J. Madeira, and B. S. Santos, "Polymeco - a polygonal mesh comparison tool," in Proc. 9th International Conference on Information Visualization IV05, pp. 842-847, IEEE CS Press, 2005.

6. B. S. Santos, S. Silva, C. Ferreira, and J. Madeira, "Comparison of methods for the simplification of mesh models of the lungs using quality indices and an observer study," in 3rd International Conference on Medical Information Visualization - Biomedical Visualization (MediVis'05), pp. 15-21, IEEE CS Press, 2005.

7. M. Botsch, S. Steinberg, S. Bischoff, and L. Kobbelt, "Openmesh - a generic and efficient polygon mesh data structure," in 1st OpenSG Symp., (Darmstadt, Germany), 2002.

8. M. Garland and P. Heckbert, "Surface simplification using quadric error metrics," in Proc. SIGGRAPH 1997, pp. 209-216, 1997.

9. C. Johnson, R. Moorhead, T. Munzner, H. Pfister, P. Rheingans, and T. S. Yoo, NIH / NSF Visualization Research Challenges Report, IEEE Press, 2006.

10. S. Silva, B. S. Santos, J. Madeira, and C. Ferreira, "Comparing three methods for simplifying mesh models of the lungs: an observer test to assess perceived quality," in Proc. SPIE 2005 vol. 5749, Image Perception, Observer Performance, and Technology Assessment, pp. 99-110, 2005.

11. M. Meyers, M. Desbrun, P. Schröder, and A. H. Barr, "Discrete differential-geometry operators for triangulated 2-manifolds," Visualization and Mathematics 3, pp. 34-57, 2002.

12. P. P. Pébay and T. J. Baker, "Analysis of triangle quality measures," Mathematics of Computation 72(244), pp. 1817-1839, 2003.

13. D. G. Johnson, Applied Multivariate Methods for Data Analysis, Duxbury, 1998.

14. V. Barnett, Sample Survey Principles and Methods, Arnold Hodder, 3rd ed., 2003.

15. S. Silva, C. Ferreira, J. Madeira, and B. S. Santos, "Perceived quality of simplified polygonal meshes: Evaluation using observer studies," in Proc. Ibero-American Symposium in Computer Graphics SIACG06, pp. 169-178, Eurographics, (Santiago de Compostela, Spain), 2006.

16. D. Hoaglin, F. Mosteller, and J. Tukey, Understanding Robust and Exploratory Data Analysis, John Wiley \& Sons, 1983.

17. "Statistica 6.0," http://www.statsoft.com, (online Mar/2006). 